\title{
INSULIN RESISTANCE, ENDOTHELIAL AND ADIPOSE DYSFUNCTION AND ATHEROSCLEROSIS
}

\author{
Gumpeny Lakshmi and Gumpeny Ramachandra Sridhar*
}

Gayatri Medical College, Visakhapatnam, India and Endocrine and Diabetes Centre, Visakhapatnam, India

\begin{abstract}
Diabetes mellitus is a multisystem group of disorders associated with insulin resistance, metabolic stress, endothelial and adipose dysfunction and accelerated atherosclerosis. As the different players leading to atherosclerosis are known, the pathogenesis and eventually targets for treatment can be identified. The purpose of this review is to update the newer aspects of pathogenic factors as well as newer putative biomarkers in atherosclerosis. Hyperglycemia causes beta cell dysfunction, which results in impaired insulin secretion, endoplasmic reticulum stress and overproduction of reactive oxygen species. Lipotoxicity, or the accumulation of increased amounts of lipids in non-adipose tissue is found in the insulin producing pancreatic beta cells impairing their function. Gluco-lipotoxicity leads to the production of inflammatory cytokines, which damage the vasculature. Endothelial dysfunction, which occurs due to all these insults can be studied by biochemical alterations and by non-invasive imaging techniques. In addition, epigenetic changes have been identified in the pathogenesis. More recent biomediators were identified to be involved in the process of atherogenesis including adiponectin, leptin, resistin, adropin, visfatin, hepatokines, bone morphogenetic protein, nerve growth factor (NGF), brain-derived neurotrophic factor (BDNF), families of micro RNAs, extracellular vesicles (exosomes, ectosomes) and a variety of environmental factors. In view of managing conventional risk factors has not prevented atherosclerotic complications, the better understanding the role of pro- and antiatherogenic factors may allow the development of novel drugs to modify them.
\end{abstract}

Adipobiology 2019; 10: 17-24

Keywords: lipotoxicity, glucotoxicity, adipokines, biomarkers, mechanotransduction, knock-out models

Received 17 November 2018, revised 29 November 2018, accepted 1 December 2018.

Correspondence: Prof. Gumpeny Ramachandra Sridhar, Director, Endocrine and Diabetes Centre, 15-12-15 Krishnanagar, Visakhapatnam, 530002, India

Email: grsridhar@hotmail.com Tel: 0891-2566301

\section{Introduction}

The biological action of insulin is initiated when it binds to the insulin receptor at the surface of target cells. This leads to the receptor being autophosphorylated, which in turn functions as a tyrosine kinase, activating two different pathways. Acting via the phosphatidylinositol 3-kinase Akt pathway it phosphorylates IRS-1 to 4, a member of the insulin receptor substrate family. The IRS contains both tyrosine phosphorylation and Ser/Thr phosphorylation sites. A balance between these two components regulates IRS function (1). Tyrosinephosphorylated IRS further binds to the adapter protein $\mathrm{P} 13 \mathrm{~K}$, activating its catalytic subunit at P13K, leading to the eventual metabolic actions of insulin.

Besides the autophosphorylation pathway, there is a separate and parallel mitogen-activated protein kinase (MAPK) pathway. Here the activation of insulin receptor leads to phosphorylation of intracellular substrate containing Shc protein, which in turn binds to the growth factor receptor, the activation of GTP exchange factor, which regulates growth and mitogenesis.

P13K/Akt primarily acts in tissues where there is rapid stimulation of glu- 
cose transport, synthesis of lipids and in energy metabolism (1). There is tissue specificity: IRS-1 proteins are associated with glucose homeostasis in adipocytes, skeletal muscle cells and pancreatic beta cells; IRS-2 in liver metabolism, and IRS-3 in adipose tissue.

Insulin resistance is associated with dysregulation of glucose, lipid and insulin regulation; cellular proliferation and apoptosis and production of gaseous signaling molecule such as nitric oxide (NO) are also disturbed (2). Vascular cell dysfunction impairs compliance, and initiates inflammation leading to atherosclerosis. Atherosclerosis results from an interplay of endothelial dysfunction, subendothelial retention of lipids, and adipose dysfunction (see below); this leads to a chronic inflammatory response which destroys the intima, resulting in vascular thrombosis and ischemia (3).

\section{Metabolic stress: glucotoxicity, lipotoxicity and gluco-lipotoxicity}

Among components of the metabolic syndrome which underlie insulin resistance, dyslipidemia, dysglycemia and obesity are crucial.

\section{Glucotoxicity}

Glucotoxicity refers to the "structural and functional damage in the pancreatic beta-cells caused by chronic hyperglycemia" (4). Elevation of glucose levels beyond physiological levels induces the expression of pro-apoptotic signals such as endoplasmic reticulum stress, oxidative stress and dysfunction of the mitochondria (4). Exposed to increased demands of insulin secretion as in hyperglycemic conditions, endoplasmic reticulum of beta cells face increased demand for synthesis of proinsulin, a precursor of insulin. This is associated with increased flux of protein through the endoplasmic reticulum. When the capacity of the endoplasmic reticulum is overwhelmed (ER stress), synthesis and delivery of proteins is impaired, an unfolded protein response is triggered to restore the balance. When the restorative capacity is exceeded, apoptotic signals to the beta cells are transmitted by stress kinases and transcription factors (MAPK, JNK, caspase-12).

In a euglycemic state, ATP-sensitive potassium channels are closed, with a release of calcium; in long standing hyperglycemic conditions, chronic release of calcium release leads to a pro-apoptopic state. Oxidative stress results in overproduction and accumulation of reactive oxygen species (ROS) and reactive nitrogen species (RNS). Reactive oxygen species are normally formed during cellular metabolism, comprising of superoxides, peroxides and hydroxyl moieties. These are neutralized by naturall antioxidants such as glutathione peroxidase, catalase, thioredoxin and superoxide dismutase. When the RNS and ROS overwhelm the native antioxidant capacity of the pancreatic beta cells, the, beta cells are damaged. Stress-induced pathways are activated leading to cellular apoptosis via nuclear factor-kB cJun N-terminal kinase 9jnk0 stress kinases and hexosamines (1).

\section{Lipotoxicity}

Lipotoxicity refers to the accumulation of increased amounts of lipids in non-adipose tissue, which leads to cellular dysfunction and death. Normally, free fatty acids (FFAs) are either synthesized de novo or are transported across the cell membrane for their physiological roles. Excess quantities of FFAs are esterified and stored in lipid droplets as triglycerides, as efficient energy stores in times of starvation. Adipose tissue is chiefly endowed with the ability to store large quantities of free fatty acids in the form of cytosolic droplets. Lipotoxicity results when excess fatty acids spill over and exceed the storage capacity of extra-adipocyte tissues. Cellular function is disturbed leading them to their death. In the pancreatic islet function is inhibited due to lipid (5). Dyslipidemia is a key component of insulin resistance ('metabolic') syndrome when excess lipids accumulate in tissues such as liver and heart, often preceding organ dysfunction. Ectopic lipid deposition in the skeletal muscle is a marker of insulin resistance. Lipid derivatives such as diacylglycerols, fatty acylCoAs and ceramide localization, composition and turnover may be the pathogenic factors (6). Besides rendering cells susceptible to apoptosis, lipotoxicity also enhances systemic low-grade inflammation (7). Other adverse effects of lipotoxicity include membrane detergent effects, impaired ability to suppress lipolysis in adjacent cells, beta oxidation of fatty acids and effects on ceramide (8). Putative pathogenic mechanisms of nonesterified fatty acids (NEFA) are summarized in Table 1

Table 1. Lipotoxic effects, potential mechanisms*

\section{Mitochondrial energy decoupling \\ Reactive oxygen species \\ Increased ceramide syntheisis \\ Activation of protein kinase C, NF-KB \\ Endoplasmic reticulum stress \\ Altered channel transporter function}

*Adapted from (9)

\section{Glucolipotoxicity}

Lipid-induced toxicity on pancreatic beta cells occurs only in the presence of hyperglycemia - a concept called glucolipotoxicity. In the presence of high glucose levels, fatty acids accumulate 
in the mitochondria; when persistent, beta cell function is impaired leading to apoptosis (1).

\section{Atherosclerosis}

While atherosclerosis essentially results from the accumulation of lipid in the subendothelial space, other contributions include mechanotransduction, inflammation in endothelial cells and the phenotypic switching of smooth muscle cells (3). Insulin resistance and endothelial dysfunction together lead to atherosclerosis and vascular disease. The endothelium, which forms the inner lining of blood vessels has multiple functions; it has been described as a paracrine, autocrine and endocrine organ responsible for vascular homeostasis $(10,11)$. It is principally influenced by insulin which affects both vasodilators (e.g. prostaglandins PG12) and vasoconstrictors (e.g. angiotensiin II). Through the activation of P13-K/Akt pathway, eNOS is phosphorylated and there is conversion of L-arginine to L-citruline and NO. The latter not only has vasodilatory roles, but has protective action on endothelium by reducing cell adhesion molecules, platelet aggregation and the synthesis of proinflammatory cytokines (10). Decreased NO can induce abnormalities found in obesity, diabetes and cardiovascular disease (12). The role of G-proteincoupled receptor kinase 2 (GRK2) in the progression of cardiovascular disease has been recognised. High levels of GRK2 may be the mediator of endothelial dysfunction by decreasing the intracellular levels of NO. In addition, advanced glycated end products (AGE) which accumulate in hyperglycemic conditions further affect the functional capacity of the blood vessels (13).

\section{Reactive oxygen species}

Reactive oxygen species have a critical role in causing endothelial dysfunction (14). Harmful ROS molecules (cf Jacob and Manoj in this volume of Adipobiology) in the vessel wall include $\mathrm{H}_{2} \mathrm{O}_{2}, \mathrm{O}_{2}, \mathrm{OH}$ and $\mathrm{ONOO}$. Hypoxia disturbs the fine balance between ROS production during mitochondrial electron transport. Instead of ROS being quenched, there is a depletion of ATP, mitochondrial depolarization, acidosis and ultimately, cell death. Besides, ROS have an adverse effect on arterial wall remodeling (14) via proliferation of smooth muscle cell and induction of inflammation. Repeated chronic mechanical stress generates free radicals which aid in adhesion of monocytes. Recent studies have shown that endothelial dysfunction is worsened by lipid peroxidation or by decreased antioxidants (15). In coronary artery disease, antioxidant enzymes are upregulated in the early stages, but they are overwhelmed by chronic exposure to ROS. Nrf2 was is a transcription factor binding to Keap1-antioxidant signal that promotes transcription of antioxidant genes (16).

\section{Endothelial dysfunction in diabetic vascular disease}

The earliest stage of endothelial dysfunction results from an impaired availability of NO. It progresses to impaired endothelium-mediated vessel regulation, poor fibrinolytic action, increased growth factor production, adhesion molecules and inflammatory genes, leading to oxidative stress and enhanced permeability (17). Pro-atherogenic properties due to endothelial dysfunction result from both a loss of its protective ability and enhanced pro-atherothrombotic properties. The endothelial dysfunction in diabetes can be summarized as follows: impaired vasodilation, increased oxidative stress, pro-coagulant and proinflammatory environment resulting in poor repair in the face of increased cellular damage (17).

\section{Clinical assessment of endothelial dysfunction}

Endothelial dysfunction was first studied by invasive methods in the coronary vasculature (12). Non-invasive procedure commonly used in clinical studies include flow-mediated dilation was used as a surrogate to assess endothelial function (18). It is safe, non-invasive, cost-effective and reproducible. Other methods include low-flow mediated constriction and endothelial peripheral arterial tonometry. While the former provides information about the resting endothelial activity, the latter provides information about changes in finger arterial pulse-wave amplitude during reactive hyperemia. Other invasive methods are venous occlusion plethysmography, which needs canulation of the brachial artery is used to measure changes in the volume of the pre-constricted vein to different substances.

Biochemical markers of endothelial function include E-selectin, ICAM-1, VCAM-1, interleukin-1, tumour necrosis factor a, interferon $\mathrm{g}$, monocyte chemoattractant protein and tissue plasminogen activator. Measurement of urinary microalbuminuria is a commonly employed, if non-specific method. Recently, asymmetrical dimethylarginine (ADMA) was shown to be a promising independent risk factor (19). ADMA, which is synthesized in the body, inhibits eNOS resulting in low NO production. Increased levels of ADMA were reported in atherosclerotic vascular disease (12).

An intriguing new marker of endothelial function has been identified: endothelial microparticles (ectosomes) (20). Circulating endothelial microparticles (EMPs, a type of extracellular vesicles) which carry biologically active compounds act as signals between different cells. They could serve as biological markers of vascular endothelial dysfunction. Levels of EMPs were higher in diabetes and correlated with diabetes related complications (20). 


\section{Obesity, inflammation and immune interactions}

Evolutionarily, survival of multicellular organisms depended on the ability to protect themselves from pathogens, to heal when attacked, and to store energy for times of need. The basic requirement was for close cross-talk between immune and metabolic systems (21). While such association was favourable in normal conditions, problems arose in conditions of metabolic stress. The adverse inflammatory conditions linked with obesity - diabetes, fatty liver and atherosclerosis occur in conditions of metabolic overload.

The inflammatory cytokine tumor necrosis factor-alpha (TNF- $\alpha$ ) was shown to be overexpressed in animal models of obesity, leading to impaired insulin action. A variety of other cytokine players were recognized at the intersection of immunity and metabolism (21): interleukins, leptin, adiponectin, visfatin, resistin, and C-reactive protein.

Which came first: obesity or inflammation? Evidence appears to point toward inflammation being the principal player resulting in obesity associated insulin resistance, hyperglycemia and dysglycemia.

\section{Recent studies on inflammatory mediators}

Resistin is an adipokine which is primarily secreted by human macrophages. Increased levels of this adipokine are associated with insulin resistance, diabetes mellitus and cardiovascular disease (22). It promotes endothelial dysfunction, proliferation of smooth muscle, inflammation in the arterial wall and endothelial dysfunction.

Adropin a newly identified protein has critical roles in energy homeostasis and in maintaining insulin sensitivity. It is a regulator of endothelial cells and can be used as a circulating biomarker. Low adropin levels were shown to be a risk factor of heart disease (23).

Visfatin, a cytokine secreted by adipose tissue could be a mediator among inflammation, endothelial dysfunction leading to atherosclerosis and to plaque instability in acute coronary syndromes. It is being evaluated as a possible plasma biomarker for inflammatory states (24).

GDF-15 is a recently recognized cytokine that is released in response to stress from a range of cells: adipocytes, macrophages, smooth muscle cells and endothelial cells. Its level increases in inflammatory conditions, and can serve as a marker of cardiovascular disease (25). It may be protective in some and pathogenic in others. GDF-15 is a promising biomarker and a potential drug target against vascular disease

Adiponectin is a long recognized anti-atherogenic adipokine which acts on the vessel wall to prevent inflammation. It modi- fies intracellular signal pathways in blood vessels (26), with insulin sensitizing properties expressed via adiponectin receptors AdipoR1 and AdipoR2. They form potential targets for pharmacologic modulation in metabolic syndrome and diabetes (27).

Hepatokines, proteins primarily secreted by the liver, influence the progression of atherosclerosis by altering endothelial function. They influence both glucose and lipid metabolism and can affect atherosclerosis by modulating inflammatory pathways. Representative hepatokines include fibroblast growth factor 21, fetuin A, selenoprotein P (28). These are important because of the increasing recognition of non-alcoholic fatty acid disease, a hepatic manifestation of metabolic syndrome which is associated with the development of type 2 diabetes mellitus and cardiovascular disease.

Bone morphogenetic protein (BMP) family plays important functions in endothelium of vessels by increasing formation of blood vessels and by modifying stress due to blood flow and oxidation. Impaired regulation of BMPs was linked with vascular disease including atherosclerosis. It influences endothelial function by acting through endothelial signaling (29).

\section{Other potential pathogenic mechanisms}

MicroRNAs (miRNAs) are non-coding RNA molecules that modulate the stability and/or the translational efficiency of target messenger RNAs (30). miRNAs were proposed to regulate gene expression following oxidative stress leading to atherosclerosis (31). Specifically the miR-221/222 cluster acts to regulate endothelial cells and maintain endothelial integrity. In atherosclerotic vessels this cluster promotes formation of neointima (32). They could also have effects on glucose and fat metabolism in other tissues.

Mechanotransduction, as mentioned earlier, refers to the pathways modulating atherogenesis because of disturbances in laminar blood flow (33). A number of pathways are described: mitogen-activated protein kinases/extracellular signal-regulated kinase 5/Kruppel-likd factor 2 signaling, extracellular signalregulated kionase/ peroxisome proliferator-activated receptor signaling. Dissecting the roles of each would help in designing therapeutic interventions (33).

Mental stress has now been recognised to accompany and to lead to insulin resistance and type 2 diabetes mellitus (34). Stress, including depression could exert their adverse effects via inappropriate formation of reactive oxygen species and the attendant downstream effects (35).

Epigenetic factors interact with transcription factors by reprogramming the transcriptome of the vascular endothelial cells (36). There could thus be involvement of epigenetic mechanisms 
in modulating endothelial injury during the atherogenic process. Air pollution is a surprising new entrant that can cause insulin resistance through its effects on inflammation, endoplasmic reticulum stress and oxidative stress (37). Being ubiquitous in urban areas, air pollutants can contribute to endothelial dysfunction associated with diabetes mellitus, in addition to other risk factors.

Human knock-outs have been receiving increasing attention in understanding the physiological role of proteins, and in designing interventions to influence their function. Human knock-outs refers to identifying 'normal' persons who lack a particular enzyme or protein, that is, without manifesting any signs or symptoms of disease. A variety of such enzyme deficiency states have been identified (38-40). These enzymes influence lipid metabolism and thereby modify the oxidative stress. Interestingly, pharmacological agents to lower the level of the enzyme ANGPTL3 $(39,41)$ was shown to lower blood lipoprotein levels with a potential for reducing the risk of atherosclerosis in an animal model, opening the way for innovative drug development in humans (41).

\section{Overview of the contributions to cell biology of atherosclerosis}

Atherosclerosis is a leading cause of disease and death in both developed and developing countries. The endothelial lining of vessels, a dynamic entity, serves as the interface with blood flow and may be considered a 'transducer of both humoral and mechanical stimuli (42). Endothelial dysfunction is triggered by biomechanical forces due to disturbed blood flow. The importance of understanding the pathogenesis is to devise preventive measures by reversing endothelial dysfunction and correcting local lipid changes.

Noteworthy, putative metabotrophic mediators acting on glucose and lipid metabolism consist of neurotrophins such as nerve growth factor (NGF) and brain-derived nerve factor (BDNF) (43). While it was originally identified to be important in neuronal growth, NGF and BDNF were shown to improve survival and activity of non-neuronal cells also, including vascular endothelium. In clinical studies of subjects with advanced metabolic syndrome (44) and with acute coronary syndromes (45), the circulating levels of NGF and BDNF were lower compared to controls.

Further, there is an intimate interaction among vascular biology, adipobiology and neuroimmunology, which has been called 'triactome' (46). It brings into focus the importance of considering the four coats of the blood vessel: intima, media, adventitia, and adiposa (perivascular adipose tissue). The "triactome" forms a potential target for the development of drugs against atherosclerosis.

Ectosomes, extracellular vesicles resulting from plasma membrane shedding, are found across life forms (47). Subjects with type 2 diabetes showed differences in the pro-angiogenic and anti-angiogenetic miRNA in ectosomes, forming potential drug targets (47). Both ectosomes and exosomes transfer signals to distant cells. While ectosomes are released from the plasma membrane, exosomes are released from multivesicular bodies (48).

Unique signaling pathways for diabetic vascular complications were explored in an effort to identify new targets for intervention. Levels of gasotransmitters such as hydrogen sulfide (48a), NO, and carbon monoxide are altered in diabetes. Traditional risk factors such as obesity, smoking and hypertension participate in reduced bioavailability of these gastrotransmitters. Modulating these is an exciting new therapeutic option, as they are also released from perivascular adipose tissue (49, 50).

The link between metabolic syndrome via endotoxins and low-grade systemic inflammation is well known (15). Interesting ways in which gut microbiota can mediate the hyperglycemic effects of organophosphate insecticides are explored (51)

Globally, tuberculosis is a common complication of diabetes needing attention. Interestingly, adipose tissue could serve as a niche storage for Mycobacterium tuberculosis. It has the potential to modify the expression of genes related to the stress response (52).

Intriguingly, BCG vaccine, traditionally used against tuberculosis infection, given as two doses to subjects with type 1 diabetes lowered glycosylated hemoglobin. The effect was seen after three years, and persisted for five years. A shift of glucose metabolism from oxidative phosphorylation to aerobic glycolysis was observed (53).

Psychological stress and disturbances of sleep lead to obesity, insulin resistance and cardiovascular morbidity $(54,55)$. Built environment is related multiple adverse effects of stress, and availability of space and time for physical exercise and relaxation. It also contributes to modifying the exposure to environmental air pollutants. All these have roles to play a role in the pathogenesis of diabetes and its complications (56-58).

Conventional risk factor management (59) has not been really helpful, as yet. Leads toward non-conventional factors, interventions targeting them, or improving the application of available modalities is necessary.

Genome wise analysis studies provide leads, but have not given the kind of transformative treatments as originally anticipated. Approaching the suspects by network analysis is the way ahead (60), along with evaluating proximate and upstream sig- 
nals emanating from the genome. Understanding the beneficial aggressive glycemic control over long-term vasculopathy is leading to role of epigenetic changes such as DNA methylation, posttranslational chromatin modifications and RNAs serving as the link between the environment and genes (61)

\section{Conclusion}

The major aim of understanding pathobiology is to prevent the occurrence of disease. Physical exercise, prevention of overweight and obesity, smoking stopping and diet control are aimed at correcting risk factors. In addition, drugs to normalize hypertension, dyslipidemia, diabetes, obesity and other complications attempt to both prevent and treat endothelial (10-13) and adipose $(43,46,48 \mathrm{a})$ dysfunction.

\section{Conflict of interest statement}

The authors certify that they have no affiliations with or involvement in any organization with any financial interest in the subject matter discussed in this review article.

\section{Acknowledgements}

We would like to thank two anonymous reviewers for their critical comments, which have helped to improve the presentation of main ideas of the present review.

\section{References}

1. Pansuria M, Xi H, Li L, Yang XF, Wang H. Insulin resistance, metabolic stress and atherosclerosis. Front Biosci (Schol Ed) 2012;4:916-931. PMID: 22202099 PMCID: PMC3319745

2. Zhao Y, Vanhoutte PM, Leung SW. Vascular nitric oxide: beyond eNOS. J Pharmacol Sci 2015;129:83-94. DOI: 10.1016/j.jphs.2015.09.002

3. Tabas I, Garcia-Cardena G, Owens GK. Recent insights into the cellular biology of atherosclerosis. J Cell Biol 2015;209:13-22. DOI: 10.1083/jcb.201412052

4. Kupsal K, Mudigonda S, Gundapaneni KK, et al. Glucotoxicity and lipotoxicity induced beta-cell apoptosis in type 2 diabetes mellitus. Int J Anal Bio-Sci 2015;3:84-9

5. Lee Y, Hirose H, Ohneda M, Johnson JH McGarry JD, Unger RH. Beta-cell lipotoxicity in the pathogenesis of non-insulin-dependent diabetes mellitus of obese rats: impairment in adipocyte-beta-cell relationships. Proc Natl Acad Sci USA 1994;91:10878-10882. PMCID: PMC45129

6. Brons C, Grunnet LG. Skeletal muscle lipotoxicity in insulin resistance and type 2 diabetes: a causal mechanism or innocent bystander? Eur J Endocrinol 2017;176:R67-78. DOI: $10.1530 /$ EJE-16-0488
7. Schaffer JE. Lipotoxicity: the many roads to cell dysfunction and cell death. J Lipid Res 2016;57:1327-1328. DOI: 10.1194/jlr.E069880

8. Tchkonia T., Corkey B.E., Kirkland J.L. Current Views of the Fat Cell. as an Endocrine Cell: Lipotoxicity. In: Bray G.A., Ryan D.H. (eds) Overweight and the Metabolic Syndrome. Endocrine Updates, 2006; vol 26. pp 105-123. Springer, Boston, MA

9. Weinberg JM. Lipotoxicity. Kidney Int 2006;70:1560-1566. DOI: $10.1038 /$ sj.ki.5001834

10. Janus A, Krupska ES, Mazur G, Doroszko A. Insulin resistance and endothelial dysfunction constitute a common therapeutic target in cardiometabolic disorders. Mediators Inflam 2016; ID:3634948. DOI:10.1155/2016/3634948

11. Taguchi K, Matsumoto T, Kobayashi T. G-protein-coupled receptor kinase 2 and endothelial dysfunction: molecular insights and pathophysiological mechanisms. J Smooth Muscle Res 2015;51:37-49. DOI: 10.1540/jsmr.51.37

12. Park KH, Park WJ. Endothelial dysfunction: clinical implications in cardiovascular disease and therapeutic approaches. J Korean Med Sci 2015;30:1213-25 DOI: 10.3346/ jkms.2015.30.9.1213

13. Altabas V. Diabetes, endothelial dysfunction and vascular repair: what should a diabetologist keep his eye on? Int J Endocrinol 2015; Article ID 848272. DOI: $10.1155 / 2015 / 848272$

14. Panth N, Paudel KR, Parajuli K. Reactive oxygen species: a key hallmark of cardiovascular disease. Adv Medicine 2016; Article ID 9152732. DOI:10.1155/2016/9152732

15. Lubrano V, Balzan S. Enzymatic antioxidant system in vascular inflammation and coronary artery disease. World $J$ Exp Med 2015;5:218-24 [DOI: 10.5493/wjem.v5.i4.218]

16. Chen B, Lu Y, Chen Y, Cheng J. The role of Nrf2 in oxidative stress-induced endothelial injuries. J Endocrinol 2015;225:R83-99. DOI:10.1530/JOE-14-0662

17. Sena CM, Pereira AM, Seica R. Endothelial dysfunction - a major mediator of diabetic vascular disease. Bioch Biophys Acta 2013;1832:2216-31 DOI: 10.1016/j.bbadis. 2013.08 .006

18. Daiber A, Steven S, Weber A, et al. Targeting vascular (endothelial) dysfunction. Br J Pharmacol 2017;174:15911619. DOI: 10.1111/bph.13517

19. Lekakis, J; Abraham, P; Balbarini, A; Blann, et al. Methods for evaluating endothelial function: a position statement from the European Society of Cardiology Working Group on Peripheral Circulation. Eur J Cardiovascular Prev Rehabil 2011;18:775-89. DOI: $10.1177 / 1741826711398179$ 
20. Deng F, Wang S, Zhang L. Endothelial microparticles act as novel diagnostic and therapeutic biomarkers of diabetes and its complications: a literature review. Biomed Res Int 2016; Article ID 9802026. DOI:10.1155/2016/9802026

21. Wellen KE, Hotamisligil GS. Inflammation, stress and diabetes. J Clin Invest 2005;115:1111-1119. DOI: 10.1172/ JCI25102

22. Park HK, Kwak MK, Kim HJ, Ahima RS. Linking resistin, inflammation, and cardiometabolic diseases. Korean J Intern Med 2017;32:239-247. DOI: 10.3904/kjim.2016.229

23. Yosaee S, Soltani S, Sekhavati E, Jazayeri S. Adropin - a novel biomarker of heart disease: a systematic review article. Iran J Public Health 2016;45:1568-1576. http://ijph. tums.ac.ir/index.php/ijph/article/view/8631

24. Hognogi LD, Simiti LV. Cardiovascular impact of visfatin - an inflammation predictor biomarker in metabolic syndrome. Clujul Med 2016;89:322-326. DOI: 10.15386/ cjmed-591]

25. Adela R, Banerjee SK. GDF-15 is a target and biomarker for diabetes and cardiovascular diseases: a translational prospective. J Diabetes Res 2015;2015: 490842. DOI: 10.1155/2015/490842

26. Balsan GA, Vieira JL, Oliveria AM, Portal VL. Relationship between adiponectin, obesity and insulin resistance. Rev Assoc Med Bras 2015;61: 72-80. DOI: 10.1590/1806-9282.61.01.072

27. Kadowaki T, Yamauchi T, Kubota N, et al. Adiponectin and adiponectin receptors in insulin resistance, diabetes and the metabolic syndrome. J Clin Invest 2006;116:1784-1792. DOI: $10.1172 /$ JCI29126

28. Yoo HJ, Choi KM. Hepatokines as a link between obesity and cardiovascular diseases. Diabetes Metab J 2015;39:1015. DOI: 10.4093/dmj.2015.39.1.10

29. Dyer LA, Pi X, Patterson C. The role of BMPs in endothelial cell function and dysfunction. Trends Endocrinol Metab 2014;25:472-480. DOI: 10.1016/j.tem.2014.05.003

30. Maagenta A, Greco S, Gaetano C, Martelli F. Oxidative stress and microRNAs in vascular disease. Int J Mol Sci 2013;14:17319-17346. DOI: 10.3390/ijms140917319

31. Zampetaki A, Dudek K, Mayr M. Oxidative stress in atherosclerosis: the role of microRNAs in arterial remodeling. Free Radic Biol Med 2013;64:69-77. DOI: 10.1016/j.freeradbiomed.2013.06.025

32. Chistiakov DA, Sobenin IA, Orekhov AN, Bobryshev YV. Human mir-221/222 in physiological and atherosclerotic vascular remodeling. Biomed Res Int 2015;2015:354517. DOI: $10.1155 / 2015 / 354517$

33. Abe J, Berk BC. Novel mechanisms of endothelial mechanotransduction. Arterioscler Thromb Vasc Biol 2014;
34:2378-2386. DOI: 10.1161/ATVBAHA.114.303428

34. Sridhar GR, Madhu K. Psychosocial and cultural issues in diabetes mellitus. Curr Sci 2002;83:1556-1564. [https:// pdfs.semanticscholar.org/d9db/ce26246b6cc40ce83c276b18f70994bff047.pdf]

35. Inoue N. Stress and atherosclerotic cardiovascular disease. J Atheroscler Thromb 2014;21:391-401. PMID: 24561512

36. Xu Y. Transcriptional regulation of endothelial dysfunction in atherosclerosis: an epigenetic perspective. J Biomed Res 2014;28:47-52. DOI: 10.7555/JBR.27.20130055

37. Rao X, Liu C, Rajagopalan S. Diabetes and metabolic syndrome. In: Nadadur SS, Hollingsworth JW, editors. Air Pollution and Health Effects. Humana Pres London. 2015; pp 213-239.

38. Slimani A, Jelassi A, Jguirim I, Najah M, Rebhi L, et al. Effect of mutations in LDLR and PCSK9 genes on phenotypic variability in Tunisian familial hypercholesterolemia patients. Atherosclerosis 2012;222:158-166. DOI:10.3109/0 0207454.2015.1057636

39. Musunuru K, Pirruccello JP, Do R, et al. Exome sequencing, ANGPTL3 mutations, and familial combined hypolipidemia. N Engl J Med 2010; 363:2220-2227. DOI: 10.1056/ NEJMoa1002926]

40. Sridhar GR, Rao AA, Srinivas K, et al. Butyrylcholinesterase in metabolic syndrome. Med Hypotheses. 2010;75:648-651. DOI: 10.1016/j.mehy.2010.08.008

41. Dewey FE, Gusarova V, Dunbar RL, et al. Genetic and pharmacologic inactivation of ANGPTL3 and cardiovascular disease. N Engl J Med 2017; 377:211-221. DOI: 10.1056/ NEJMoa1612790

42. Tabas I, Cardena GG, Owens GK. Recent insights into the cellular biology of atherosclerosis. J Cell Biol 2015;209:1322. DOI: $10.1083 /$ jcb.201412052

43. Yanev S, Aloe L, Flore M, Chaldakov GN. Neurotrophic and metabotrophic potential of nerve growth factor and brain-derived neurotrophic factor: linking cardiometabolic and neuropsychiatric diseases. World J Pharmacol 2013;2:92-99. DOI:10.5497/wjp.v2.i4.92

44. Chaldakov GN, Fiore M, Stankulov IS, Manni L, Hristova MG, Antonelli A, Ghenev PI, Aloe L. Neurotrophin presence in human coronary atherosclerosis and metabolic syndrome: a role for NGF and BDNF in cardiovascular disease. Progr Brain Res 2004;146:279-289. DOI 10.1016/ S0079-6123(03)46018-4

45. Manni L, Nikolova V, Vyagova D, Chaldakov GN, Aloe L. Reduced plasma levels of NGF and BDNF in patients with acute coronary syndromes. Int J Cardiol 2005;102:169-171. DOI: 10.1016/j.ijcard.2004.10.041 
46. Chaldakov GN, Flore M,, Ghenev PI, Beltowski J, Rancic G, Tuncel N, Aloe L. Triactome: neuro-immune-adipose interactions. Implications in vascular biology. Front Immunol 2014;5: [Article 1301] DOI:10.3389/fimmu.2014.00130

47. Stepien EL, Durak-Kozica M, Kaminska A, TargoszKorecka, Libera M, Tylko G, et al. Circulating ectosomes: determination of angiogenic microRNAs in type 2 diabetes. Theranostics 2018;8:3874-3890. DOI: 10.7150/ thno. 23334

48. Lawson C, Vicencio JM, Yellon DM, Davidson SM. Microvesicles and exosomes: new players in metabolic and cardiovascular disease. J Endocrinol 2016;228:R57-R71 DOI: 10.1530/JOE-15-0201

48a. Bełtowski J, Wójcicka G, Jamroz-Wiśniewska A. Hydrogen sulfide in the regulation of insulin secretion and insulin sensitivity: Implications for the pathogenesis and treatment of diabetes mellitus. Biochem Pharmacol 2018;149:60-76. DOI: 10.1016/j.bcp.2018.01.004

49. Evans JL, Goldfine ID. A new road for treating the vascular complications of diabetes: so let's step on the gas. Diabetes 2018;65:346-348 [doi.org/10.2337/dbi15-0029]

50. Kennedy S, Salt IP. Molecular mechanisms regulating perivascular adipose tissue - potential pharmacological targets? Br J Pharmacol 2017;174:3385-3387. DOI:10.1111/ bph.13969

51. Velasquez MT. Altered gut microbiota: a link between diet and the metabolic syndrome. Metab Synd Rel Dis 2018; DOI:10.1089/met.2017.0163

52. Bompadre MB, Montagna G, Kuhl AA, Lozza L, Weiner J, Kupz A, et al. Mycobacterium tuberculosis infection modulates adipose tissue biology. PLoS Pathog 2017;13: e10006676.https://doi.org/10.1371/journalppat.1006676

53. Kuhtreiber WM, Tran L, Kim T, Dybala M, Nguyen B,
Plager S, et al. Long-term reduction in hyperglycemia in advanced type 1 diabetes: the value of induced aerobic glycolysis with BCG vaccinations. Npj Vaccines 2018;3:23. DOI:10.1038/s41541-018-0062-8

54. Bot I, Kuiper J. Stressed brain, stressed heart? Lancet 2017;389:770-771. DOI: 10.1016/S0140-6736(17)30044-2

55. Sridhar GR, Sanjana NSN. Sleep, circadian dysrhythmia, obesity and diabetes. World J Diab 2016; 7: 515-522. DOI: 10.4239/wjd.v7.i19.515

56. Sridhar GR, Sudhir Kumar P, Venkata P, Appa Rao A, Vijay Kishore Durai, Madhu K, et al. Built environment factors, psychosocial factors and diabetes mellitus: A south Indian study. Ind J Clin Med 2010:1 15-22. DOI:10.4137/IJCM. S4710

57. Yang L, Griffin S, Khaw KT, Wareham N, Panter J. Longitudinal associations between built environment characteristics and changes in active commuting. BMC Public Health 2017;17:458 DOI:10.1186/s12889-017-4396-3

58. Bowe B, Xie Y, Li T, Yan Y, Xian H, Al-Aly Z. The 2016 global and national burden of diabetes mellitus attributable to PM2-5 air pollution. Lancet Planet Health 2018;2:e30112 [doi.org/10.1016/S2542-5196(18)30140-2]

59. Barrett EEJ, Liu Z, Khamaisi M, King GL, Klein R, Klein BEK, et al. Diabetic microvascular disease: an Endocrine Society Scientific statement. J Clin Endocrinol Metab 2017;102:1-68 DOI:10.1210/jc.2017-01922

60. Mei H, Li L, Griswold M, Mosley T. Gene expression metaanalysis of seven candidate gene sets for diabetes traits following a GWAS pathway study. Front Genetics 2018;9:52 DOI:10.3389/fgene.2018.00052

61. De Rosa S, Arcidiacono B, Chiefari E, Brunetti A, Indolfi C, Foti DP. Type 2 diabetes mellitus and cardiovascular links. Front Endocrinol 2008;9:2. DOI:10.3389/fendo.2018.00002 